\title{
Patterns of Root Morphology of Mandibular Third Molars and Their Clinical Implications in a Cohort of Sri Lankan Patients
}

\author{
Nadeena Swarnagupta Jayasuriya ${ }^{1,}$, , Irosha Rukmali Perera ${ }^{2,}$, Sakuntha Ratnapreya ${ }^{3}$, \\ Fhelen Debbie Da Costa ${ }^{4}$ \\ ${ }^{1}$ Department of Oral and Maxillofacial Surgery, Faculty of Dental Sciences, University of Peradeniya, Peradeniya, Sri Lanka \\ ${ }^{2}$ Preventive Oral Health Unit, National Dental Hospital (Teaching), Sri Lanka, Colombo, Sri Lanka \\ ${ }^{3}$ Department of Dental Surgery, Asiri Surgical Hospital, Colombo, Sri Lanka \\ ${ }^{4}$ College of Dental Sciences, Davangere, Karnataka, India
}

\section{Email address:}

nadeenaja@yahoo.com (N. S. Jayasuriya), irosha_rukmali@yahoo.com (I. R. Perera), sakuntha.left.ratnapriya@gmail.com (S. Ratnapreya), fhe10dec@gmail.com (F. D. D. Costa)

${ }^{*}$ Corresponding author

\section{To cite this article:}

Nadeena Swarnagupta Jayasuriya, Irosha Rukmali Perera, Sakuntha Ratnapreya, Fhelen Debbie Da Costa. Patterns of Root Morphology of Mandibular Third Molars and Their Clinical Implications in a Cohort of Sri Lankan Patients. International Journal of Clinical Oral and Maxillofacial Surgery. Vol. 7, No. 1, 2021, pp. 6-12. doi: 10.11648/j.ijcoms.20210701.12

Received: May 25, 2021; Accepted: June 7, 2021; Published: June 21, 2021

\begin{abstract}
Background: Mandibular third molar surgeries garnered recognition as one of the common treatment procedures provided by Oral \& Maxillofacial practices across the globe. Mandibular third molars are not only impacted, often giving rise to bothering issues to patients ranging from pain to difficulty in opening mouth but create challenges in their surgical removal. Postoperative complications of those surgeries such as pain, swelling, trismus and alveolar osteitis (dry socket) could be prevented and controlled by meticulous preoperative assessment of tooth-related and patient-related factors. Pattern of root morphology could be one important tooth- related factor connected to difficulties and subsequent postoperative complications of mandibular third molar surgeries. Aim: Against this backdrop, the aim of this study was to explore the patterns of root morphology of mandibular third molars and to elucidate their associations with selected attributes of the surgeries, self-reported preoperative pain and selected postoperative complications among a cohort of Sri Lankan patients. Materials \& Methods: A hospital based, descriptive cross-sectional study was conducted among 715 patients (represented wider age range from adolescents to older adults) who underwent surgical removal of mandibular third molars at the Department of Oral \& Maxillofacial Surgery, Faculty of Dental Sciences, University of Peradeniya, Sri Lanka. Of extensive socio-demographic, clinical and radiological investigation data collected, those data on root morphology and numbers of roots, inferior dental canal relationship, self-reported preoperative pain, duration of the surgery, flap design, tooth sectioning and postoperative pain and swelling complications were used for the present analysis. Data entry and analysis was done using SPSS-21 Statistical Software Package. Results: Based on our findings on patterns of root morphology, straight two roots were the most common (34.4\%) across all age groups, followed by convergent two- roots $(19.3 \%)$ fused straight- roots (16.3\%) and distally curved two- roots (13.8) among Sri Lankan patients. Almost half (46.8\%), of patients had inferior dental nerve canal placed away from mandibular third molar roots. Moreover, root morphology was significantly associated with presence of preoperative pain, duration of the surgery as well as occurrence of postoperative swelling $(\mathrm{p}<0.05)$. Conclusions: Patterns of root morphology of mandibular third molars may implicate on certain processes of their surgical removal and its outcomes. Therefore, careful preoperative assessment of root morphology and related factors of those teeth becomes useful in tailored patient care for minimal postoperative complications, better patient experience and quality-of-life.
\end{abstract}

Keywords: Mandibular Third Molars, Impaction, Surgical Removal, Root Morphology, Interdental Canals, Postoperative Complications, Quality-of-Life 


\section{Introduction}

Mandibular third molars (wisdom teeth) are often impacted demonstrating a frequency of occurrence ranging from $18 \%$ to $32 \%$ with geographical variations $[1,2]$. Nevertheless, the global prevalence of impacted mandibular third molars considered to be $24.40 \%$ ( $95 \%$ confidence interval [ $95 \% \mathrm{CI}]$ : $18.97 \%$ to $30.80 \%$ based on a systematic review and meta-analysis [3]. Despite usual eruption of mandibular third molars at ages ranging from 16 to 24 -years, could be partially or completely impacted according to literature [5]. Consequently, pathological changes comprising infection, pain, caries and root resorption could occur indicating surgical removal of mandibular third molars [4]. Therefore, majority of patients accessing Oral \& Maxillofacial Surgery practices for wisdom tooth surgeries across the globe comprise of late adolescents and young adults [5]. However, symptomatic impacted mandibular third molars could bother middle aged adults as well [6].

Despite the need, surgical removal of mandibular third molars is fraught with an array of postoperative complications such as pain, swelling, trismus and infection [7]. Moreover, those surgeries often pose the challenges of potential risk of causing transient or even permanent disturbance to the inferior alveolar nerve with the reported incidence ranging from $1.3 \%$ to $5.3 \%$ [8]. Therefore, the probability of this complication is determined by the proximity of the impacted third molar tooth to the inferior alveolar canal thus elevating the incidence of possible disturbance up to $19 \%$ [9]. Coronectomy has become the preferable surgical modality in this regard [10]. Therefore, careful preoperative radiological assessment of tooth-related factors such as type of impaction (soft tissue vs bony impaction), impaction depth, angulation, relationship to interdental canals, ramus relationship and root morphology has become fundamental to planning mandibular third molar surgeries. Accordingly, Mesioangular impactions were identified as the type of angulation that was more closely placed to the inferior alveolar canal whilst interruption of the white line was recognized as the most reliable risk predictor sign [11]. Hence, forecasting difficulties and possible complications preoperatively provide many opportunities for customizing the surgical techniques in impacted mandibular third molar removal. Root morphology patterns demonstrating geographic variations [12] seem salient tooth-related factor that could impact on those surgeries. For example, tooth sectioning procedures in the surgery could be influenced by the pattern of root morphology [13]. Divergent root patterns need tooth sectioning more often compared to other root patterns. Against this backdrop, we aim to explore and expound the patterns of root morphology of mandibular third molars and their associations with selected attributes of surgical procedures, preoperative pain and selected postoperative complications.

\section{Materials \& Methods}

\subsection{Study Design}

A hospital based, descriptive cross-sectional study was conducted.

\subsection{Study Setting}

The Department of Oral \& Maxillofacial Surgery, Faculty of Dental Sciences, University of Peradeniya, Sri Lanka was the study setting.

\subsubsection{Study Participants}

Patients who underwent mandibular third molar surgeries during the study period, willing to participate comprised the study participants, The sample size was calculated by the formula estimating a single proportion with a requirement for 95\% CI and 5\% Standard Error [14] with estimated prevalence of any postoperative complication as $50 \%$ as there were no previous studies in this regard in Sri Lankan context. The calculated minimum sample size was 384, subsequently adjusted for $10 \%$ non-response as 438 . However, final sample included 715 participants. Informed consent was obtained from the participants before inclusion into the study.

\subsubsection{Data Collection}

The data collection period including patient follow up, was from 20/05/2016 to 01/05/2018. Data were collected by a pre-tested, validated questionnaire and data extraction from clinical and radiological investigation records. Trained data collectors performed the data collection. Socio-demographic information, self-reported symptoms were collected by a self-administered component of the questionnaire. Data were collected during pre, peri and postoperative follow-up phases of surgical removal of mandibular third molars.

Present analysis involved data on preoperative self-reported pain, patterns of root morphology of mandibular third molar, relationship to interdental canal, duration of surgery, flap design, tooth sectioning, pain and swelling experienced by patients one week postoperatively.

\subsubsection{Data Entry and Analysis}

As there were 10 patterns of root morphology, they were aggregated into 2 broad categories as "straight two-rooted" and "all other types" that included divergent, convergent, curved, fused and 3-rooted root types. Duration of the surgical procedure was dichotomized as " $\leq 45$ minutes" and "> 45 minutes' Flap design was categorized as "No flap", "Envelope flap" and "two or three sided flap". Further, tooth sectioning was categorized as "No", "Once" and "Twice or more.

Ethics approval for the present study was obtained from the Ethics Review Committee of Faculty of Dental Sciences, University of Peradeniya, Sri Lanka. Data were entered and analyzed using SPSS-21 Statistical Software Package. Frequency distributions were used as descriptive statistics 


\section{Results \& Discussion}

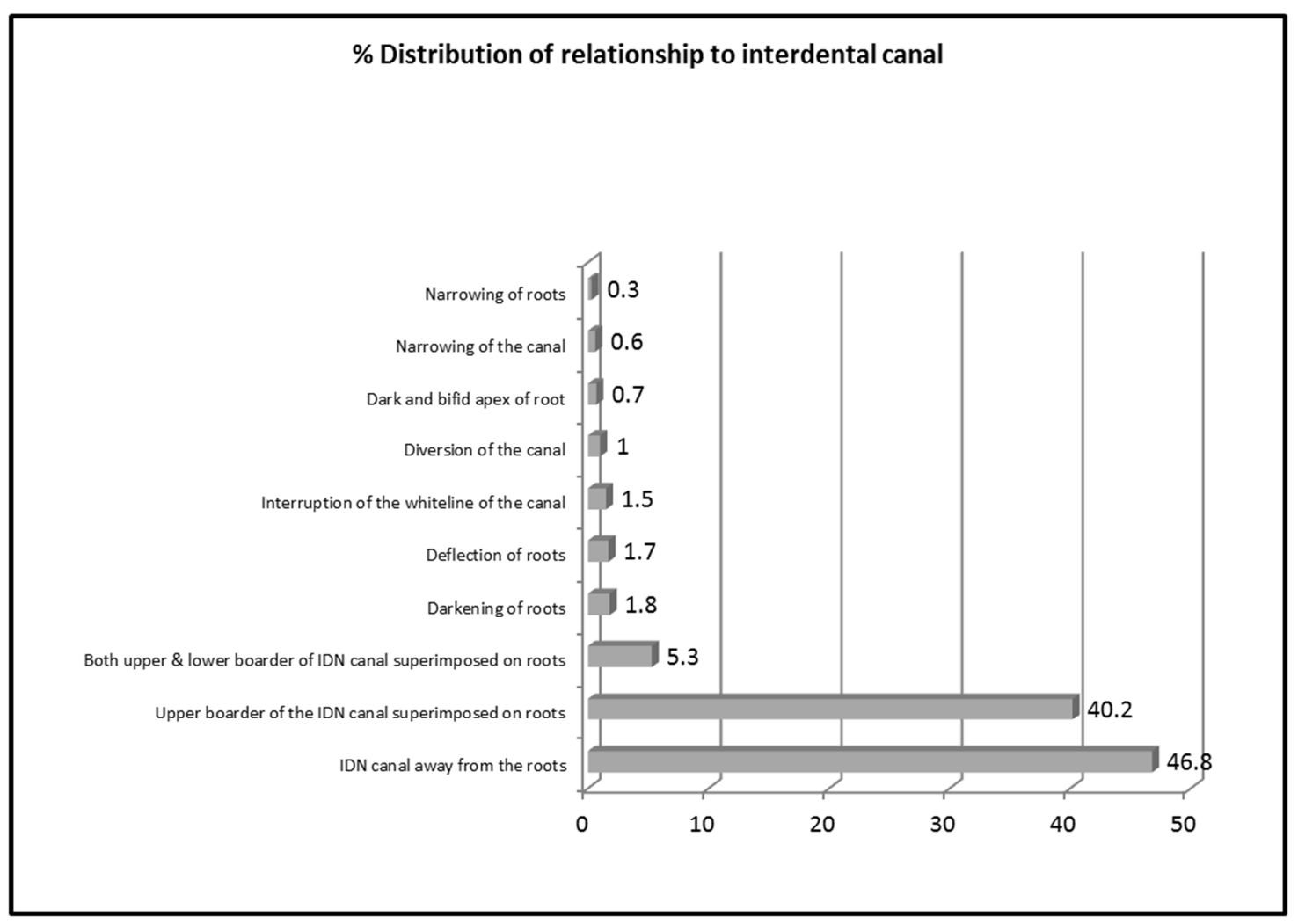

Figure 1. Distribution of Categories of Relationship to Inferior Dental Canals.

\subsection{Relationship to Inferior Dental Canal and Clinical Implications}

"Figure 1" illustrates the distribution of categories of relationship to inferior dental canals among 711 patients with relevant data who underwent mandibular third molar surgeries. Accordingly, radiological findings of the majority (46.8\%) of patients demonstrated positioning of inferior dental nerve (IDN) canal away from roots, whilst another 40.2\% demonstrated upper boarder of the IDN canal superimposed on roots of mandibular third molars. Furthermore, 5.3\% showed radiological findings of both upper and lower boarders of IDN canal superimposed on roots. In addition, darkening of roots, deflection of roots and interruption of white line of the canal were evident among $1.8 \%, 1.7 \%$ and $1.5 \%$ of patients respectively.

\subsection{Pattern of Root Morphology and Its Association with Surgical Processes}

Table 1 illustrates the distribution of patients by patterns of root morphology pertaining to 710 patients with relevant data. Accordingly, straight two-roots was the most common root morphological pattern detected among $34.4 \%$ of patients followed by convergent two-roots (19.3\%), fused straight roots $(16.3 \%)$ and distally curved two roots $(13.8 \%)$. Further, divergent two roots were evident among $4.2 \%$ of patients. Of fused variants, distally curved fused type dominated (3.7\%) followed by mesially curved fused roots $(1.4 \%)$. However, three-rooted non fused teeth occurred only among $0.8 \%$ of patients. Therefore, as emerged from the findings, heterogenous patterns of root morphology of mandibular third molar teeth were evident in the present cohort of Sri Lankan patients.

Table 1. Pattern of root morphology of mandibular third molars among Sri Lankan patients.

\begin{tabular}{ll}
\hline Root Morphology & N (\%) \\
\hline Straight two-rooted & $244(34.4)$ \\
Divergent two-rooted & $30(4.2)$ \\
Convergent two-rooted & $137(19.3)$ \\
Distally curved two-rooted & $98(13.8)$ \\
Mesially curved two-rooted & $29(4.1)$ \\
Fused Straight & $116(16.3)$ \\
Fused curved distally & $26(3.7)$ \\
Fused curved mesially & $16(1.4)$ \\
Three-rooted non fused & $6(0.8)$ \\
One root curved other straight & $14(2.0)$ \\
Total & $710(100.0)$ \\
\hline
\end{tabular}

Table 2 describes the association of root morphology with selected attributes of respective mandibular third molar surgeries. They included self- reported preoperative pain, duration of surgery, flap design, tooth sectioning, pain and swelling one week postoperatively. 
As shown in Table 2, higher proportion of patients with other root morphologies of their mandibular third molars, reported preoperative pain, underwent $>45$ minutes duration of surgeries and reported swelling one-week postoperatively compared to those who had straight two- rooted mandibular third molars. Those differences were statistically significant ( $\mathrm{p}$ $<0.05$ ). Therefore, patterns of root morphology of mandibular third molars of current cohort of patients who underwent their surgical removal was significantly associated with presence of preoperative pain, duration of the surgery and swelling reported one week postoperatively, However, flap design and tooth sectioning of surgical procedure and pain reported one-week postoperatively were not significantly associated with the patterns of root morphology $(p>0.05)$.

There is limited research published in literature exploring the patterns of root morphology of mandibular third molars and their implications on processes and outcomes of their surgical removal. As revealed by the findings of our study, $99.1 \%$ of mandibular third molars of Sri Lankan patients were two-rooted. These findings differed from that of a study on anatomy of third molars [15]. Accordingly, 77\% of mandibular third molars were two-rooted, 5\% were three-rooted and another $1 \%$ had four roots. In our study, only $0.8 \%$ was three-rooted and four roots were not reported. In contrast, our findings corroborated a study conducted among Brazilian population on anatomy of mandibular third molars [16]. Findings of that study revealed the most common pattern was two roots $(98.3 \%)$, Further, the same study reported conical simple type $(88.9 \%)$, with absence of laceration $(89.2 \%)$, divergence (84\%), and fusion (80\%) [16]. However, in our study, occurrence of fusion was only $22.3 \%$ and divergence was as low as $4.2 \%$. Moreover, similar study conducted among Korean population revealed $56 \%$ of mandibular third molars with two roots and $37.9 \%$ with a single root [12]. Therefore, substantiated by such evidence, it is rational to suggest presence of geographic and population specific heterogeneity in patterns of root anatomy and morphology of mandibular third molars. Further studies warranted in this regard in light of epidemiological and clinical significance of patterns of root morphology of mandibular third molars.

Table 2. Association of root morphology with preoperative pain and selected surgical processes and postoperative complications.

\begin{tabular}{|c|c|c|c|c|}
\hline Attribute & Straight two-roots & Other root types & Chi-Square value & p-value \\
\hline Preoperative pain & $\mathrm{N}(\%)$ & $\mathrm{N}(\%)$ & & \\
\hline No & $140(57.4)$ & $217(46.6)$ & & \\
\hline Yes & $104(42.6)$ & $249(53.4)$ & & \\
\hline Total & $244(100.0)$ & $466(100.0)$ & 7.487 & $0.006 * *$ \\
\hline Duration & N (\%) & $\mathrm{N}(\%)$ & & \\
\hline$\leq 45$ minutes & $228(93.4)$ & $395(84.8)$ & & \\
\hline$>45$ minutes & $16(6.6)$ & $71(15.2)$ & & \\
\hline Total & $244(100.0)$ & $466(100.0)$ & 11.218 & $0.001 * *$ \\
\hline Flap design & $\mathrm{N}(\%)$ & $\mathrm{N}(\%)$ & & \\
\hline No flap & $66(28.6)$ & $144(33.7)$ & & \\
\hline Envelope flap & $133(57.6)$ & $233(54.6)$ & & \\
\hline Other & $32(13.8)$ & $50(11.7)$ & & \\
\hline Total & $231(100.0)$ & $427(100.0)$ & 2.043 & 0.360 \\
\hline Tooth sectioning & N (\%) & $\mathrm{N}(\%)$ & & \\
\hline None & $132(54.1)$ & $289(62.0)$ & & \\
\hline Once & $66(27.0)$ & $98(21.0)$ & & \\
\hline Twice or more & $46(18.9)$ & $79(17.0)$ & & \\
\hline Total & $244(100.0)$ & $466(100.0)$ & 4.534 & 0.104 \\
\hline Postop pain* & $\mathrm{N}(\%)$ & $\mathrm{N}(\%)$ & & \\
\hline No & $93(48.7)$ & $184(54.3)$ & & \\
\hline Yes & $98(51.3)$ & $155(45.7)$ & & \\
\hline Total & $191(100.0)$ & $339(100.0)$ & 0.219 & 0.216 \\
\hline Postop swelling* & $\mathrm{N}(\%)$ & $\mathrm{N}(\%)$ & & \\
\hline No & $130(68.4)$ & $264(77.9)$ & & \\
\hline Yes & $60(31.6)$ & $75(22.1)$ & & \\
\hline Total & $190(100.0)$ & $339(100.0)$ & 5.727 & $0.017 * *$ \\
\hline
\end{tabular}

${ }^{*}$ one week postoperatively $* * \mathrm{p}<0.05$.

Duration of third molar surgery could be considered as an indicator of its relative complexity. Supporting this notion, our findings revealed significantly longer duration of surgeries involving mandibular third molars having complex root morphologies compared to straight roots. Interestingly, it has been attempted to develop and validate a statistical model to predict third molar surgery operative time [17]. In this study, the sample comprised of 150 participants who had 450 third molars removed. The index sample had 100 patients having 313 third molars extracted with a mean age of $25.4 \pm 10.0$ years.
The mean extraction time was $6.4 \pm 7.0$ minutes. The multiple linear regression models included third molar location, Winter's classification, tooth morphology, number of teeth extracted, procedure type, and surgical experience. Moreover, statistically significant differences were not observed between the index sample and the validation sample $(n=50$, teeth=137) for any of the study variables. As this study included both maxillary and mandibular third molar extractions among young adults and due to other salient methodological differences, it is not possible to directly compare the findings 
with our study. Nevertheless, many studies corroborated those key findings and one study classified the predictors of third molar surgical difficulties as demographic, anatomic and operative [18] However, tooth morphology which includes root morphology emerged as a predictor of surgery time with an array of other predictors [18] supporting the core premise of our study.

Furthermore, flap design has garnered recognition as a significant factor in surgical removal of impacted mandibular third molars that allows optimal visibility and access [19]. Moreover, flap designs influences the severity of postoperative complications [19] and subsequent healing of surgically created defect [20]. Many studies therefore, have reported novel methods of flap designs other than commonly used envelope, two-sided and three sided flaps [19-21]. However, there are remaining information gaps on impact of root morphology of mandibular third molars on flap designs.

Tooth sectioning is commonly employed as a surgical technique in impacted third molar surgeries, Therefore, novel methods of tooth sectioning has been introduced by Oral \& Maxillofacial Surgeons. Accordingly, a modified method of tooth sectioning between distal root and the remainder of the tooth for horizontally impacted teeth found to be efficiently eliminating the resistance from the bone and adjacent second molar tooth thus enabling single sectioning most frequently for a relatively safe and straight forward surgery [22]. The anatomy of roots therefore, could play a discernible role in tooth sectioning. However, our findings did not demonstrate significant associations of root morphology with flap design or tooth sectioning. This could be attributed to dichotomous categorization of tooth morphology patterns as straight two roots vs other types. Perhaps a more detailed breakdown of ten patterns of root morphology among our study participants could have provided different findings. Therefore, further studies warranted in this regard having even larger cohorts of patients,

Our findings demonstrated a significant association of root morphology of mandibular third molars with preoperative self-reported pain and swelling reported one week postoperatively. Accordingly, significantly higher proportion of patients who had root morphologies other than straight two-root type reported pain and swelling preoperatively and postoperatively respectively. Those findings could plausibly be attributed to possible complicated patterns of root morphology of impacted mandibular molars becoming more symptomatic. Furthermore, their surgical removal lasted for longer durations could have contributed for postoperative swelling. However, as there is an array of other factors related to the surgery such as bone removal, density of bone, techniques of local anaesthesia, age and of the patient, oral hygiene status, life-style related risk habits of patients, existing medical conditions, type of impaction, angulation assessed by Winter's Classification, operator factors, use of anti-inflammatory medication such as steroids that could influence the incidence of postoperative swelling, further explorations needed in this regard [22-25] for more conclusive findings.
Postoperative swelling denotes a common complication of third molar surgeries having biological and social impact [5, 22]. Therefore, prediction of postoperative facial swelling subsequent to mandibular third molar extractions received attention of researchers. Accordingly, artificial neural network model has been developed incorporating predictors of patient-personal factors, anatomical factors of mandibular third molars and factors of surgical procedure [23]. This model demonstrated $98 \%$ accuracy in predicting postoperative swelling following mandibular third molar surgeries. Number of roots of mandibular third molar was included into this model among an array of other predictors [23]. Moreover, there is research evidence to suggest age as a primary risk factor not only for difficulties in third molar surgeries but for occurrence of postoperative complications [25]. Accordingly, $>25$ years appeared to be significantly associated with higher incidence of third molar extraction complications [25]. In addition, pre-existing infection such as periodontitis around third molar was a significant predictor of inflammatory third molar surgery complications [26]. Hence, the predictors of those complications were classified as demographic, health status, pathologic and anatomic by researchers [23-28]. Therefore, it is prudent to argue that the significant association of root morphology pattern of mandibular third molar with preoperative pain and postoperative swelling in our study plausibly may indicate existed pathology and inflammation that could be related to complicated root morphologies. Further, longer surgical procedures have implicated for increased frequency of postoperative complications of mandibular third molar surgeries [27, 28]. Hence, our findings could have corroborated this finding possibly mediated via root morphology. However, further research warranted in this regard.

Patient-centered outcome measures in oral surgery were explored way back in 2003 [29]. Clinical outcomes often assessed by clinicians may not exactly reflect the impact of a given oral surgical procedure for the quality-of-life as perceived by the patients in physical, psychological and social dimensions [29]. Interestingly, the prospective cohort study conducted in this regard included patients who underwent third molar surgeries [30]. Significant deterioration of perceived quality- of- life among patients who underwent third molar surgeries was evident at immediate postoperative period compared to preoperative status which improved significantly in 1, 3 and 6-months postoperatively [30]. However, preoperative pericoronitis was significantly associated with patient perceived quality-of-life [30]. Moreover, a subsequent systematic review and meta-analysis revealed the first day postoperative as the time for highest negative impact for quality-of-life of patients who underwent third molar surgeries [31]. Minimal negative impact to quality-of-life immediately and across postoperative trajectory therefore, would enhance patient experience and satisfaction. Moreover, a recent study suggested the effectiveness of modified triangular flap over conventional envelope flap in terms pain, wound dehiscence and trismus 3-days postoperatively contributing to better quality-of-life for patients [32]. Against this backdrop, root 
morphology patterns and related factors of mandibular third molars teeth needing surgical removal seem demonstrating many important implications for Oral \& Maxillofacial clinical practice. Those implications range from customized patient awareness in obtaining their consent for the surgery to customized patient care, optimal outcomes and better qualityof- life for patients.

\section{Conclusions}

Patterns of mandibular third molar root morphology were significantly associated with preoperative pain, duration of the surgery and reporting of swelling one week postoperatively among present cohort of Sri Lankan patients. Our findings provided new insights into the unequivocal importance of careful preoperative assessment of patterns of root morphology and related factors of mandibular third molars awaiting surgical removal. However, there are many confounding factors that could influence preoperative pain, duration of the surgery and consequent postoperative swelling connected to or independent of root morphology. Notably, soft tissue vs bony impactions, impaction depth, existing pericoronitis, medical conditions, oral hygiene status, age of the patient and complexity of surgical techniques are important in this regard as aforementioned. Hence, further follow up studies are recommended with mulvariate analyses accounting for those confounders. Furthermore, in depth classification of root morphology patterns such as divergent, convergent, curved, fused and three-rooted are recommended in future studies. Complimented by patient-related and operator-related factors, pre-operative assessment of root morphology patterns becomes fundamental to providing tailored patient care for minimal postoperative complications and better patient experience of quality-of-life. In order to fill the remaining information gaps on associations of root morphology patterns with surgical processes and outcomes among different population groups, future research recommended in this regard preferably with age and gender stratified samples.

\section{Acknowledgements}

Authors wish to acknowledge Dr PWST Premarathne, Dr PC Senevirathne, Dr HSMB Herath, Dr Prakrama Wijekoon, Dr Kanchana Kapugama and Prof. Manjula Attygalla for their contributions.

\section{References}

[1] Kalai Selvan S, Ganesh SKN, Natesh P, Moorthy MS, Niazi TM, Babu SS. (2020). Prevalence and Pattern of Impacted Mandibular Third Molar: An Institution-based Retrospective Study. J Pharm Bioallied Sci. 2020; 12 (Suppl 1): S462-S467.

[2] Alfadil L, Almajed E. (2020) Prevalence of impacted third molars and the reason for extraction in Saudi Arabia. Saudi Dent J. Jul; $32 \quad$ (5): 262-268. doi: 10.1016/j.sdentj.2020.01.002.
[3] Carter K, Worthington S. (2016). Predictors of Third Molar Impaction: A Systematic Review and Meta-analysis. J Dent Res. Mar; 95 (3): 267-76. doi: 10.1177/0022034515615857.

[4] Niedzielska IA, Drugacz J, Kus N, Kreska J. (2006). Panoramic radiographic predictors of mandibular third molar eruption. Oral Surg Oral Med Oral Pathol Oral Radiol Endod. 2006; 102: 154-8.

[5] Renton T, Wilson NH. (2016). Problems with erupting wisdom teeth: signs, symptoms, and management. British Journal of General Practice. 2016 Aug 1; 66 (649): e606-8.

[6] Samsudin AR, Mason DA. (1994). Symptoms from impacted wisdom teeth. Br J Oral Maxillofac Surg. 1994; 32 (6): 380-383.

[7] Bailey E, Kashbour W, Shah N, Worthington HV, Renton TF, Coulthard P. (2020). Surgical techniques for the removal of mandibular wisdom teeth. Cochrane Database Syst Rev.; 7 (7): CD004345.

[8] Bui CH, Seldin EB, Dodson TB (2003). Types, frequencies, and risk factors for complications after third molar extraction. J Oral Maxillofac Surg.; 61: 1379-89.

[9] Sedaghatfar M, August MA, Dodson TB. (2005). Panoramic radiographic findings as predictors of inferior alveolar nerve exposure following third molar extraction. J Oral Maxillofac Surg.; 63: 3-7.

[10] Martin A, Perinetti G, Costantinides F, Maglione M (2015).. Coronectomy as a surgical approach to impacted mandibular third molars: a systematic review. Head \& face medicine. Dec; 11 (1): 1-1.

[11] Deshpande, P., V Guledgud, M., \& Patil, K. (2013). Proximity of impacted mandibular third molars to the inferior alveolar canal and its radiographic predictors: a panoramic radiographic study. Journal of maxillofacial and oral surgery, 12 (2), 145-151. https://doi.org/10.1007/s12663-012-0409-z.

[12] Park JB, Kim N, Park S, Ko Y. (2013). Evaluation of number of roots and root anatomy of permanent mandibular third molars in a Korean population, using cone-beam computed tomography. Eur J Dent. Jul; 7 (3): 296-301. doi: 10.4103/1305-7456.115413.

[13] Liao R, Jiang X, Wang R, Li X, Zheng Q, Huang H. (2020). Removal of Horizontally Impacted Mandibular Third Molars with Large Root Bifurcations Using a Modified Tooth Sectioning Method. J Oral Maxillofac Surg. 2021 Apr; 79 (4): 748-755. e1. doi: 10.1016/j.joms.2020.12.011.

[14] Lwanga, Stephen Kaggwa, Lemeshow, Stanley \& World Health Organization. (1991). Sample size determination in health studies: a practical manual / S. K. Lwanga and S. Lemeshow. World Health Organization. https://apps.who.int/iris/handle/10665/40062.

[15] Sidow SJ, West LA, Liewehr FR, Loushine RJ. (2000). Root canal morphology of human maxillary and mandibular third molars. J Endod. Nov; 26 (11): 675-8. doi: 10.1097/00004770-200011000-00011.

[16] Da Silva Sampieri MB, Viana FLP, Cardoso CL, Vasconcelos MF, Vasconcelos MHF, Gonçales ES. Radiographic study of mandibular third molars: evaluation of the position and root anatomy in Brazilian population. Oral Maxillofac Surg. 2018 Jun; 22 (2): 163-168. doi: 10.1007/s10006-018-0685-y. 
[17] Susarla SM, Dodson TB. (2013). Predicting third molar surgery operative time: a validated model. J Oral Maxillofac Surg. Jan; 71 (1): 5-13. doi: 10.1016/j.joms.2012.08.004.

[18] Susarla SM, Dodson TB. (2005). Estimating third molar extraction difficulty: a comparison of subjective and objective factors. J Oral Maxillofac Surg. Apr; 63 (4): 427-34. doi: 10.1016/j.joms.2004.12.003.

[19] Rabi A, Haris PMM, Panickal DM, Ahamed S, Pulikkottil VJ, Haris KTM. (2017). Comparative Evaluation of Two Different Flap Designs and Postoperative Outcome in the Surgical Removal of Impacted Mandibular Third Molar. J Contemp Dent Pract. Sep 1; 18 (9): 807-811. doi: 10.5005/jp-journals-10024-2131.

[20] Hassan B, Al-Khanati NM, Bahhah H. (2020). Effect of lingual-based flap design on postoperative pain of impacted mandibular third molar surgery: Split-mouth randomized clinical trial. Med Oral Patol Oral Cir Bucal. Sep 1; 25 (5): e660-e667. doi: 10.4317/medoral.23666.

[21] Menziletoglu D, Guler AY, Basturk F, Isik BK, Erdur EA.(2020). Comparison of two different flap designs for bilateral impacted mandibular third molar surgery. J Stomatol Oral Maxillofac Surg. Sep; 121 (4): 368-372. doi: 10.1016/j.jormas.2019.08.006.

[22] Liao R, Jiang X, Wang R, Li X, Zheng Q, Huang H. (2021). Removal of Horizontally Impacted Mandibular Third Molars with Large Root Bifurcations Using a Modified Tooth Sectioning Method. J Oral Maxillofac Surg. 2021 Apr; 79 (4): 748-755. e1. doi: 10.1016/j.joms.2020.12.011.

[23] Zhang, W., Li, J., Li, Z. B., \& Li, Z. (2018). Predicting postoperative facial swelling following impacted mandibular third molars extraction by using artificial neural networks evaluation. Scientific reports, 8 (1), 12281. https://doi.org/10.1038/s41598-018-29934-1.

[24] Larrazábal C, García B, Peñarrocha M, Peñarrocha M. (2010). Influence of oral hygiene and smoking on pain and swelling after surgical extraction of impacted mandibular third molars. J Oral Maxillofac Surg. Jan; 68 (1): 43-6. doi: 10.1016/j.joms.2009.07.061.
[25] Chuang SK, Perrott DH, Susarla SM, Dodson TB. (2007). Age as a risk factor for third molar surgery complications. J Oral Maxillofac Surg. 2007 Sep; 65 (9): 1685-92. doi: 10.1016/j.joms.2007.04.019.

[26] Chuang SK, Perrott DH, Susarla SM, (2008). Dodson TB. Risk factors for inflammatory complications following third molar surgery in adults. J Oral Maxillofac Surg. Nov; 66 (11): 2213-8. doi: 10.1016/j.joms.2008.06.067.

[27] Blondeau F, Daniel NG. (2007). Extraction of impacted mandibular third molars: postoperative complications and their risk factors. J Can Dent Assoc. 2007 May; 73 (4): 325. PMID: 17484797.

[28] Baqain ZH, Karaky AA, Sawair F, Khraisat A, Duaibis R, Rajab LD. (2008). Frequency estimates and risk factors for postoperative morbidity after third molar removal: a prospective cohort study. J Oral Maxillofac Surg. Nov; 66 (11): 2276-83. doi: 10.1016/j.joms.2008.06.047. Erratum in: J Oral Maxillofac Surg. 2009 Mar; 67 (3): 706. Khaisat, Ameen [corrected to Khraisat, Ameen]. PMID: 18940492.

[29] McGrath C, Comfort MB, Lo EC, Luo Y. (2003). Patient-centred outcome measures in oral surgery: validity and sensitivity. Br J Oral Maxillofac Surg. Feb; 41 (1): 43-7. doi: 10.1016/s0266-4356(02)00289-9.

[30] McGrath C, Comfort MB, Lo EC, Luo Y. (2003). Can third molar surgery improve quality of life? A 6-month cohort study. J Oral Maxillofac Surg. Jul; 61 (7): 759-63; discussion 764-5. doi: 10.1016/s0278-2391(03)00150-2.

[31] Duarte-Rodrigues L, Miranda EFP, Souza TO, de Paiva HN, Falci SGM, Galvão EL. (2018). Third molar removal and its impact on quality of life: systematic review and meta-analysis. Qual Life Res. Oct; 27 (10): 2477-2489. doi: 10.1007/s11136-018-1889-1.

[32] Simșek Kaya G, Yapıcı Yavuz G, Saruhan N. (2019). The influence of flap design on sequelae and quality of life following surgical removal of impacted mandibular third molars: A split-mouth randomised clinical trial. J Oral Rehabil. 2019 Sep; 46 (9): 828-835. doi: 10.1111/joor.12814. 